\title{
Invasion patterns of metastatic high-grade serous carcinoma of ovary or fallopian tube associated with BRCA deficiency
}

\author{
M Carolina Reyes ${ }^{1}$, Angela G Arnold ${ }^{2}$, Noah D Kauff ${ }^{2,3,4}$, Douglas A Levine ${ }^{3,4}$ and \\ Robert A Soslow ${ }^{1,4}$
}

${ }^{1}$ Department of Pathology, Memorial Sloan-Kettering Cancer Center, New York, NY, USA; ${ }^{2}$ Clinical Genetics Service, Department of Medicine, Memorial Sloan-Kettering Cancer Center, New York, NY, USA; ${ }^{3}$ Gynecology Service, Department of Surgery, Memorial Sloan-Kettering Cancer Center, New York, NY, USA and ${ }^{4}$ Weill Cornell Medical College, New York, NY, USA

\begin{abstract}
High-grade serous carcinomas of the uterine adnexa with BRCA1 deficiency (high-grade serous carcinomas$B R C A)$ have recently been described to demonstrate characteristic histopathological features. We hypothesize that metastatic high-grade serous carcinomas-BRCA cases exhibit characteristic morphological features as well. We studied 102 high-grade serous carcinomas with known BRCA1 and BRCA2 genotype from the archives of the Department of Pathology at Memorial Sloan-Kettering Cancer Center. The primary site morphological characteristics of these cases were reported previously; we now focus solely on tumor morphology in sites other than the uterine adnexa (ie, metastatic sites). The study group consisted of the following case types: 13 BRCA1 germline mutations; 5 BRCA1 somatic mutations; 10 BRCA1 promoter methylation; 4 BRCA2 germline mutations; 1 BRCA2 somatic mutation; 11 lacking BRCA1 or BRCA2 abnormality; 58 cases lacking BRCA1 or BRCA2 germline mutation. Two observers independently scored invasion patterns and microscopic tumor architecture while blinded to genotype. Concordance between observers and correlations between metastatic patterns and the following indices were studied: genotype, primary site tumor characteristics, and BRCA1 immunohistochemistry. Concordance between observers was excellent ( $\kappa$ values $>0.9$ ). All cases with BRCA1 or 2 abnormalities showed either pushing pattern metastases (76\%) or infiltrative metastases composed only of micropapillae (24\%). In contrast, all cases lacking BRCA1 or 2 abnormalities showed infiltrative metastases that contained combinations of papillary, glandular, and, rarely, cribriform and micropapillary architecture $(P<0.0001$ for comparison with pushing metastasis and $\boldsymbol{P}<\mathbf{0 . 0 0 1}$ for comparison with purely micropapillary architecture). Morphological assessment of metastatic carcinomas, a highly reproducible exercise, accurately correlated with BRCA1 status in every case, unlike morphological assessment of primary site adnexal high-grade serous carcinomas or BRCA1 immunohistochemistry. Metastatic high-grade serous carcinomas-BRCAs exhibit characteristic morphological features that appear more sensitive and specific for BRCA mutations than two other morphologically based prediction systems and should be easier to apply in practice. These findings should be validated prospectively in an independent cohort.

Modern Pathology (2014) 27, 1405-1411; doi:10.1038/modpathol.2013.237; published online 28 February 2014
\end{abstract}

Keywords: BRCA; metastasis; ovarian carcinoma; serous carcinoma; transitional cell carcinoma

High-grade serous carcinomas of the uterine adnexa with BRCA1 deficiency (high-grade serous carcinoma-BRCA) have recently been described to demonstrate characteristic histopathological features

Correspondence: Dr RA Soslow, MD, Department of Pathology, Memorial Sloan-Kettering Cancer Center, 1275 York Avenue, New York, NY 10065, USA.

E-mail: gynbreast@mskcc.org

Received 28 August 2013; accepted 1 November 2013; published online 28 February 2014 reminiscent of BRCA1-associated breast carcinoma with medullary-like features, ${ }^{1-4}$ including solid and transitional cell-like growth patterns, ${ }^{5}$ a high mitotic index, ${ }^{5,6}$ numerous tumor infiltrating lymphocytes, 5,6 and severe nuclear atypia., 5 These features have been described not only in the setting of BRCA1 germline mutation, but also with somatic mutations and BRCA1 promoter methylation. ${ }^{5}$ A detailed study of these features from our group ${ }^{5}$ focused on tumor features in the primary site, the uterine adnexa. The current investigation focuses on tumor features in 
metastatic sites, specifically, the peritoneum. Our interest in this subject stems from a study that reported an association between ovarian transitional cell carcinoma, round peritoneal metastases, and relatively favorable survivals, which were thought to be at least in part attributable to high rates of optimal peritoneal debulking in affected patients. ${ }^{7}$ These features may also be associated with BRCA mutations. We hypothesize that metastatic highgrade serous carcinomas- $B R C A$ cases exhibit similar characteristic morphological features.

\section{Materials and methods}

We studied 44 high-grade serous carcinomas with known BRCA1 and BRCA2 genotype from the archives of the Department of Pathology at Memorial Sloan-Kettering Cancer Center. The primary site morphological characteristics of these cases were reported previously, ${ }^{5}$ and focused solely on tumor morphology in sites other than the uterine adnexa (ie, metastatic sites). An additional 58 cases lacking a BRCA1 or BRCA2 germline mutation were also included for study, yielding 102 cases in total. Only cases with slides representing primary staging and debulking procedures were included for study. All cases were FIGO stage III or IV.

Slides from the archives of the Department of Pathology at Memorial Sloan-Kettering Cancer Center were retrieved after obtaining Institutional Review Board approval. Twenty-five cases had been included as part of The Cancer Genome Atlas pilot project, ${ }^{8}$ whereas 9 BRCA1 germline cases were drawn from a series of sequential patients who underwent genetic testing as part of clinical care at Memorial SloanKettering Cancer Center between 2008 and 2010. The Cancer Genome Atlas, a multi-institutional effort sponsored by the National Cancer Institute and the National Human Genome Research Institute, catalogs genomic alterations responsible for cancer using multiple integrated high-throughput genome analysis methods. Techniques used include gene expression profiling, copy number assessment, SNP genotyping, genome-wide DNA methylation profiling, microRNA profiling, and whole exome sequencing. High-grade serous carcinoma is one of the tumor types recently completed by The Cancer Genome Atlas. Quality control for all The Cancer Genome Atlas specimens is accomplished through a biospecimen core resource to ensure tumor cellularity and nucleic acid integrity. The distribution of genetic abnormalities is provided in Table 1.

The mean number of slides per case from metastatic sites was 12 , with the minimum number of slides being 5. Diagnosis as high-grade serous carcinoma was based upon Gilks' modification to the WHO criteria for diagnosing high-grade serous carcinoma. ${ }^{9,10}$ Gilks' modification classifies highgrade ovarian carcinomas as high-grade serous carcinomas for cases showing solid, cribriform,
Table 1 Distribution of genetic abnormalities

\begin{tabular}{lc}
\hline Genetic subgroups & $\begin{array}{c}\text { Number of cases } \\
(\mathrm{n}=102)\end{array}$ \\
\hline BRCA1-associated cases & 28 \\
BRCA1 germline mutation & 13 \\
BRCA1 somatic mutation & 5 \\
BRCA1 promoter methylation & 10 \\
BRCA2-associated cases & 5 \\
BRCA2 germline mutation & 4 \\
BRCA2 somatic mutation & 1 \\
No BRCA abnormality & 11 \\
No germline BRCA mutation & 58 \\
\hline
\end{tabular}

microcystic, and transitional cell-like architectural patterns as long as confirmatory endometrioid features such as squamous metaplasia, endometrioid adenofibroma, associated endometriosis, and synchronous endometrioid carcinoma of endometrium are absent. The kinship between high-grade ovarian transitional cell carcinoma and high-grade serous carcinoma has been explored in several recent publications. ${ }^{11-13}$

Each metastatic site was evaluated for tumor architecture (Figure 1). Solid architecture was defined as tumor cells arranged in sheets. Cribriform or 'pseudoendometrioid' architecture was defined as tumor cells growing in a back-to-back glandular and nested pattern with punched out micro-lumens. Transitional cell-like architecture was defined as tumor forming broad papillae with thick, stratified layers of tumor cells. Solid, pseudoendometrioid, and transitional cell-like architecture have been referred to as 'SET' architecture in a previous publication from our group. ${ }^{5}$ Papillary tumors showed tumor cells arranged around finger-like vascular connective tissue. Purely micropapillary tumors had dyshesive clusters of tumor cells lacking a central vascular core surrounded by a retraction space; hierarchically branched papillae and micropapillae with slit-like spaces and transitions from nested to micropapillary architecture were not classified as 'micropapillary' for the purposes of this work. Tumors with a pushing/circumscribed invasive pattern had well-defined and smooth borders at the interface between tumor and surrounding tissue, whereas irregularly infiltrative tumors had a jagged interface at the tumor's periphery (Figure 2). We adjudicated problematic cases by asking ourselves whether blunt or sharp dissection would have been necessary to excise the metastasis; sharp dissection was equated with irregularly infiltrative tumors. Each slide was scored as having either a pushing/ circumscribed or irregularly infiltrative invasive pattern. When multiple metastases from the same patient demonstrated different invasive patterns, the pattern found in the most slides was recorded as the predominant pattern. Slides from metastases that did not demonstrate the interface between tumor and surrounding stroma were not scored. 

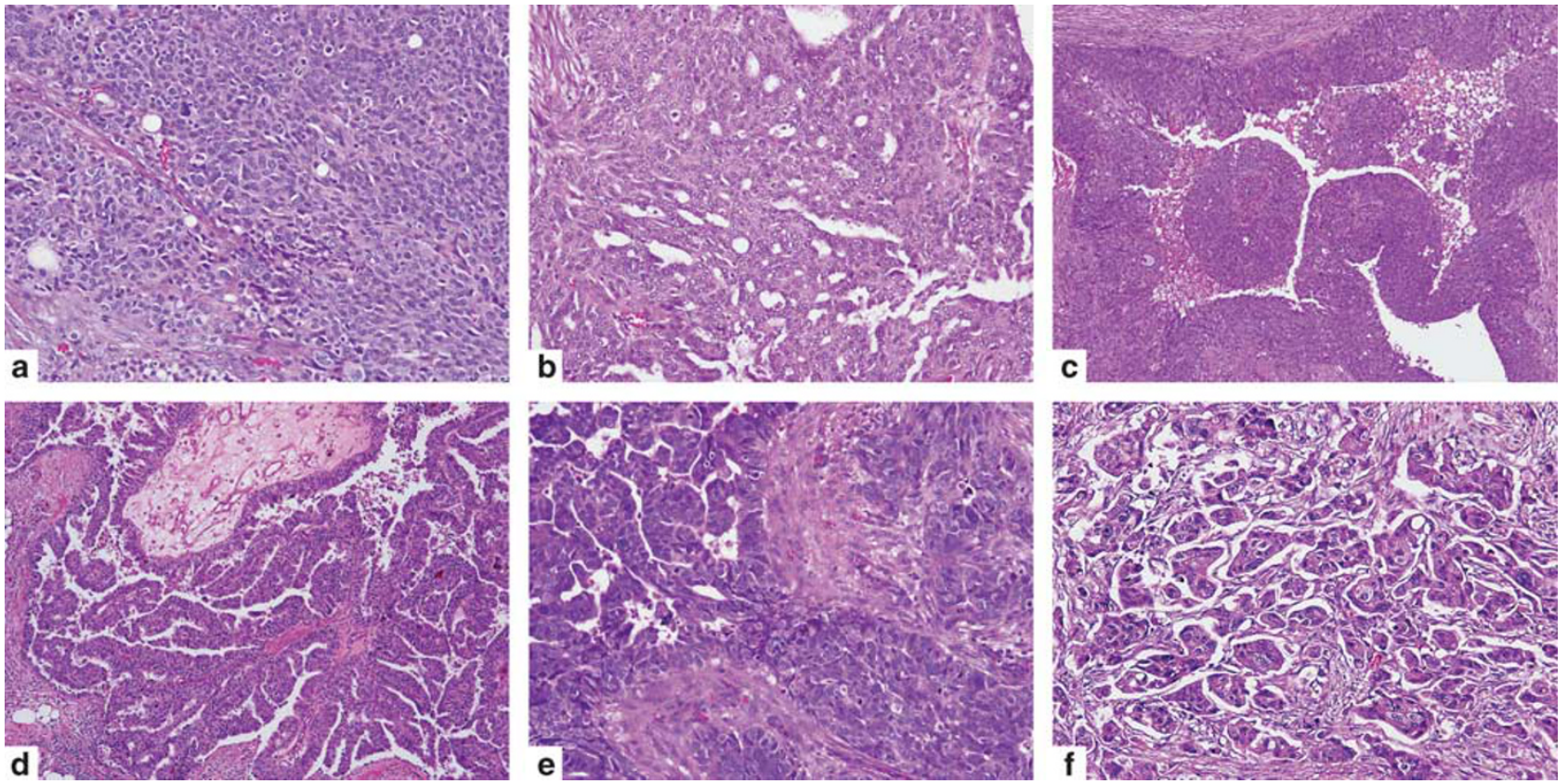

Figure 1 Tumor architecture: (a) solid, (b) pseudo-endometrioid, (c) transitional cell-like, (d) papillary and micropapillary, (e) solid and micropapillary, and (f) purely micropapillary.

Two study pathologists (MCR and RAS) reviewed each case independently without knowledge of genotype. Concordance values were calculated for micropapillary architecture and pushing versus infiltrative invasive patterns based on review of cases with a full repertoire of known BRCA abnormalities $(n=44)$.

The morphology of these tumors in the primary site (ovary or fallopian tube) $)^{5}$ and BRCA1 immunophenotype $^{14}$ have been reported previously; this information was then correlated on a case-by-case basis with the morphology of matched metastases.

\section{Results}

The number of peritoneal metastatic sites ranged from 1 to 9, with an average of 4 per case. Metastatic sites included omentum, falciform ligament, sigmoid serosa, appendix, pelvic side wall, paracolic gutter, bladder serosa, and cul-de-sac.

Both study pathologists agreed on the presence of purely micropapillary architecture in all cases but one, a BRCA1 germline case. (We agreed that micropapillary architecture was present in 7 of 44 cases and disagreed in 1 of 44 cases; $\kappa=0.92$.) We also agreed on the assessment of pushing versus infiltrative invasive patterns in every case but 2 . (We agreed that pushing invasion was present in 23 of 44 cases and disagreed in 2 of 44 cases; $\kappa=0.91$.) In one case, a BRCA germline case, one observer diagnosed a pushing pattern of invasion and in another case, a BRCA unaffected case, the same observer diagnosed an infiltrative invasive pattern. Given the high levels of concordance, we arbitrarily chose one pathologist's diagnoses for statistical analysis going forward.

All cases with BRCA1 or 2 abnormalities showed either pushing pattern metastases $(76 \%)$ or infiltrative metastases composed only of micropapillae $(24 \%)$. In contrast, all cases lacking BRCA1 or 2 abnormalities showed infiltrative metastases containing combinations of architectures: papillary, glandular, and, rarely, pseudoendometrioid and micropapillary. These data are summarized in Table 2.

Purely micropapillary architecture was found exclusively in cases with BRCA1 or 2 alterations, 8 of 33 cases $(24 \%)$ versus 0 of 69 cases $(0 \%$ $(P<0.001))$. Considering only tumors from patients with germline BRCA1 mutation revealed a similar trend, 5 of 13 cases (38 versus $0 \%(P=0.0002)$ ). Purely micropapillary metastases mostly had an infiltrative invasive pattern. In two BRCA1 germline patients, there were both metastases with micropapillary architecture and an infiltrative invasion pattern, as well as non-micropapillary architecture with a pushing invasive pattern. All other cases coded as micropapillary architecure contained only an infiltrative invasive pattern.

Pushing/circumscribed invasion patterns were exclusively found in tumors with BRCA1 or 2 abnormality, 25 of 33 cases $(76$ versus $0 \%$ $(P<0.0001))$. Considering only tumors from patients with germline BRCA1 mutation revealed a similar trend, 10 of 13 cases $(77$ versus $0 \%(P<0.0001)$ ). Nine of 33 tumors demonstrated at least one metastatic focus with a pushing pattern and one with an infiltrative pattern; as stated in the Materials and Methods, we scored cases using the 

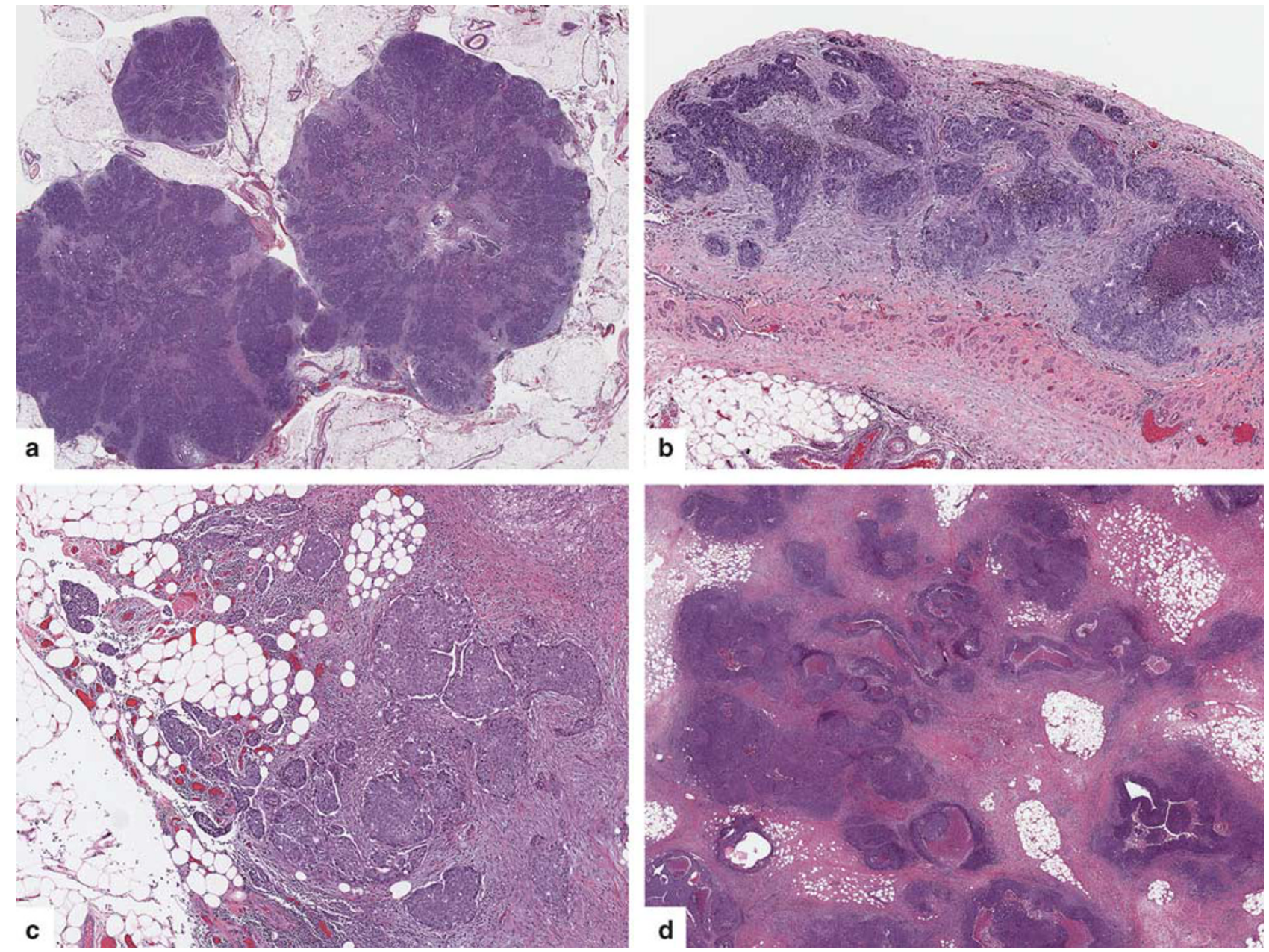

Figure 2 Invasion patterns: (a) small and round metastases, (b) pushing metastasis lacking an infiltrative border, (c and d) infiltrative metastases. We adjudicated problematic cases by asking ourselves whether blunt or sharp dissection would have been necessary to excise the metastasis; sharp dissection was equated with irregularly infiltrative tumors.

predominant pattern of invasion. The pushing pattern predominated in every case but one.

Metastases with pushing/circumscribed patterns of invasion mostly showed combinations of solid, pseudoendometrioid, and transitional cell architecture (SET architecture), with occasional cases demonstrating papillary architecture. Parenthetically, SET architectural patterns in primary ovarian highgrade serous carcinomas are often seen in tumors with BRCA abnormalities. ${ }^{5}$ Overall, metastases with infiltrative invasion patterns showed papillary and/ or micropapillary architecture, with occasional cases exhibiting pseudoendometrioid architecture.

With only five exceptions, all cases coded as pushing metastases $(n=25)$ were associated with primary adnexal tumors with SET architecture and with only three exceptions, all infiltrative metastases with BRCA abnormalities $(n=19)$ were associated with primary adnexal tumors that lacked SET architecture, irrespective of BRCA status. One of the exceptional cases had SET architecture in the adnexa and was categorized as showing infiltrative metastases, but this case had metastases that were difficult to classify because of the presence of some features of a pushing invasive pattern.

We correlated metastatic site invasive growth patterns with the morphology-based algorithmic assessment of BRCA1 status based on primary site characteristics, previously described by Soslow et al. ${ }^{5}$ This algorithm incorporates evaluation of the predominant architectural growth pattern (with emphasis on SET patterns), mitotic index, and presence of geographic necrosis. A related algorithm, with very similar predictive power, incorporates evaluation for SET patterns, tumor infiltrating lymphocytes, and presence of geographic necrosis. The algorithms stratify cases into BRCA-negative, $B R C A$-positive, and BRCA-indeterminate categories. According to the first algorithm described above, four of the tested BRCA1-associated cases were indeterminate morphologically and two were called BRCAnegative (only one BRCA1 germline mutated case was called indeterminate; all other indeterminate and negative cases either had BRCA1 promoter methylation or a somatic BRCA1 mutation). Evaluation of metastases from these cases showed pushing invasive 
Table 2 Distribution of tumor architecture and patterns of invasion

\begin{tabular}{|c|c|c|c|}
\hline Genetic subgroup & Tumor architecture & Patterns of invasion & Number of cases \\
\hline \multirow[t]{4}{*}{ BRCA1 germline } & & & 13 \\
\hline & Solid & Pushing & 5 \\
\hline & Papillary & Pushing & 4 \\
\hline & Micropapillary & Infiltrating & 4 \\
\hline \multirow[t]{3}{*}{ BRCA1 somatic } & & & 5 \\
\hline & Cribriform, solid & Pushing & 4 \\
\hline & Micropapillary & Infiltrating & 1 \\
\hline \multirow[t]{4}{*}{ BRCA1 promoter methylation } & & & 10 \\
\hline & Cribriform, pseudoendometrioid & Pushing & 5 \\
\hline & Solid & Pushing & 4 \\
\hline & Micropapillary & Infiltrating & 1 \\
\hline \multirow{3}{*}{ BRCA2 germline } & & & 4 \\
\hline & Papillary & Pushing & 3 \\
\hline & Micropapillary & Infiltrating & 1 \\
\hline \multirow[t]{2}{*}{ BRCA2 somatic } & & & 1 \\
\hline & Micropapillary & Infiltrating & 1 \\
\hline \multirow[t]{3}{*}{ No BRCA abnormality } & & & 11 \\
\hline & Papillary & Infiltrating & 7 \\
\hline & Cribriform, pseudoendometrioid & Infiltrating & 4 \\
\hline \multirow[t]{4}{*}{ No germline BRCA mutation } & & & 58 \\
\hline & Papillary & Infiltrating & 25 \\
\hline & Cribriform, pseudoendometrioid & Infiltrating & 15 \\
\hline & Solid & Infiltrating & 18 \\
\hline
\end{tabular}

patterns or micropapillary architecture, features not encountered in high-grade serous carcinomas lacking a BRCA abnormality. Two of the tested BRCA1unassociated cases were called BRCA-positive on morphologic examination of the primary site, but each had metastases composed of combinations of infiltrative invasive pattern with papillary and micropapillary architectures, which was not encountered in the $B R C A$-associated metastases.

We also correlated metastatic site growth patterns with BRCA1 immunohistochemistry, as published previously. ${ }^{14}$ Loss of BRCA1 expression was found in all tumors from patients with BRCA1 germline mutations and in almost all cases with tumors having somatic BRCA1 mutation or promoter methylation. Each of the cases with paradoxically retained BRCA1 staining (one promoter methylation case and three somatic mutation cases) had characteristic BRCA-high-grade serous carcinoma metastatic patterns; three had pushing invasive patterns and one had a micropapillary architecture with infiltrative pattern. Retention of BRCA1 staining was found in all cases lacking a BRCA abnormality and, again, none of these cases had $B R C A$-high-grade serous carcinoma metastatic patterns. Therefore, morphological assessment of metastatic carcinomas accurately correlated with BRCA1 status in every case, unlike morphological assessment of primary site adnexal high-grade serous carcinomas or BRCA1 immunohistochemistry.

\section{Discussion}

Our results confirm the observations of Kommoss' group $^{7}$ that linked transitional cell-like architecture to the presence of rounded metastatic deposits and we expand on them by ascertaining an association between BRCA genotype and this phenotype. We show that $B R C A$-high-grade serous carcinomas demonstrate characteristic growth patterns in metastatic sites: deposits with rounded and pushing contours, similar to triple-negative breast carcinomas with medullary-like features, or infiltrative deposits composed exclusively of micropapillae with retraction spaces. Cases with rounded and pushing metastasis mostly displayed SET patterns in both primary and metastatic sites, whereas cases with infiltrative metastasis usually lacked SET patterns in primary and metastatic sites. Certain morphologic features have been shown to predict an association with BRCA aberration. $B R C A 1$-associated high-grade serous carcinomas more frequently have solid, pseudoendometroid, and transitional morphology, higher mitotic indices, geographic necrosis, and tumor infiltrating lymphocytes. ${ }^{5}$ In other words, the presence of SET architecture, particularly when tumor infiltrating lymphocytes are numerous, is a clue that the tumor might have a BRCA abnormality. In this study, metastases from BRCA-unassociated high-grade serous carcinomas were always infiltrative, but unlike BRCA-high-grade serous carcinomas, the deposits were composed of combinations of cellular nests, cribriform glands, papillae with hierarchical and micropapillary branching, and infiltrative micropapillae with retraction spaces. As suggested by Kommoss' group, ${ }^{7}$ it is possible that the rate of optimal tumor debulking in patients with $B R C A$-high-grade serous carcinomas, ${ }^{15}$ one of the most important prognostic indicators, ${ }^{16-18}$ might be favorably influenced by the prevalence of 
Table 3 Sensitivities and specificities for predicting BRCA abnormalities using primary tumor morphology and BRCA1 immunohistochemistry

\begin{tabular}{lcc}
\hline & Sensitivity & Specificity \\
\hline Primary tumor morphology $^{\mathrm{a}}$ & 1.0 & 0.57 \\
BRCA1 IHC $^{\mathrm{b}}$ & 0.89 & 0.88 \\
\hline
\end{tabular}

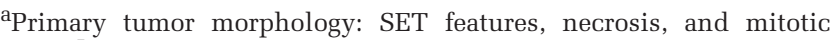
index. $^{5}$

${ }^{\mathrm{b}}$ BRCA1 IHC: BRCA1 immunohistochemistry. ${ }^{14}$

metastases that are not irregularly infiltrative into soft tissue. Should these patterns be recognizable on radiologic imaging studies, they could be used for preoperative assessments that stratify patients to be treated either with optimal tumor debulking surgery (for pushing metastases or a subset of infiltrative metastasis) or neoadjuvant chemotherapy (infiltrative metastasis).

With the description of these findings, there are now three morphologically based methods that can be applied to determine which high-grade serous carcinomas are most likely to have a BRCA1 mutation or BRCA1 promoter methylation; germline genetic testing can then be performed in appropriate cases. The three methods are: evaluation of adnexal primary site high-grade serous carcinomas using one of the algorithms published by Soslow et al; ${ }^{5}$ BRCA1 immunohistochemical study; ${ }^{14}$ and examination of metastatic high-grade serous carcinoma growth patterns. The first method, although highly sensitive for detecting BRCA1 abnormality, is not specific, and the second method makes use of an immunohistochemical antibody that can be difficult to optimize and results in staining patterns that are difficult to interpret (see Table 3 for comparison of sensitivities and specificities of the two previous studies). The third method, described herein, appears at least as robust as BRCA1 immunohistochemical staining, and more easily applied in general practice settings.

It appears that assessment for the presence of pushing invasive pattern metastases and pure micropapillary architecture is highly reproducible, but there were rare cases that were interpreted differently by the two observers. One of two cases with disagreement about the presence of a pushing pattern of invasion demonstrated confluent nodules of metastatic carcinoma that in some cases entrapped fat; this was ultimately scored as infiltrative and correlated with the absence of a $B R C A$ abnormality. Another morphologic pattern that could lead to difficulty in classification is minimal, microscopic infiltration into fat at the margin of a metastasis that is otherwise circumscribed and appears to have a pushing pattern at low-power magnification (Figure 3). This was observed in one case, from a patient with germline BRCA1 mutation, and was interpreted as 'pushing pattern of invasion'

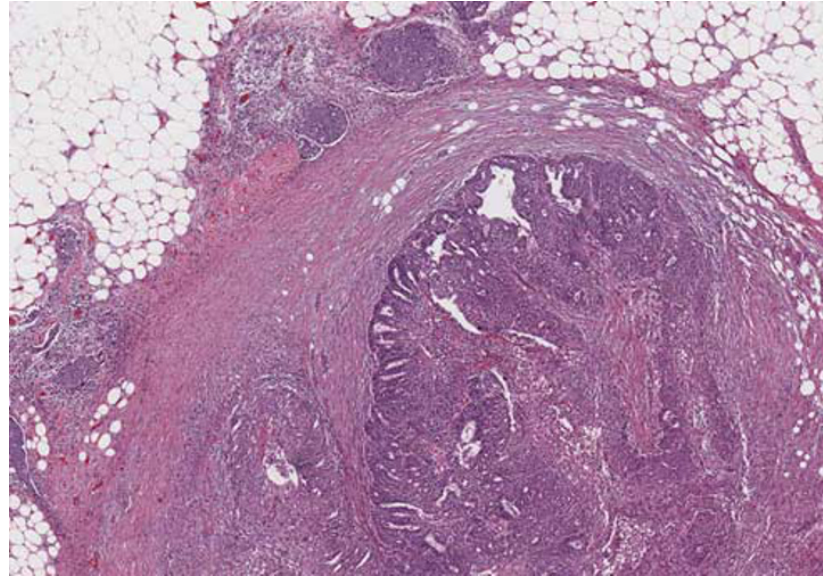

Figure 3 Pushing invasion pattern with infiltration at the periphery.

by both observers. It should be mentioned that only metastases with an evaluable tumor/stromal interface were scored for pushing versus infiltrative invasion. This rule did not lead to the exclusion of any case from the study, suggesting that this should not introduce difficulties in the practical application of the criterion, but in four cases it did exclude several metastatic sites from evaluation. These cases were ultimately adjudicated based on the prevalent invasive pattern in metastases with evaluable tumor/stromal interfaces. Although there was only one case where the observers disagreed about the presence of purely micropapillary architecture, this criterion is conceptually more complex than the pushing criterion and unfamiliarity with the "pure micropapillary architecture' emphasized here could theoretically lead to misclassification. To reiterate, purely micropapillary tumors had dyshesive clusters of tumor cells lacking a central vascular core surrounded by a retraction space (Figure 2). This pattern is identical to the pattern of invasion that is so characteristic of invasive low-grade serous carcinoma, although the nuclear grade is obviously different. Hierarchically branched papillae and micropapillae with slit-like spaces and transitions from nested to micropapillary architecture were not classified as 'micropapillary' in this study and nearperfect correlation with BRCA1 abnormality supports the biological distinctiveness of these patterns.

Although this study provides data that are potentially important from diagnostic, prognostic, and therapeutic perspectives, there are some shortcomings. First, morphologic features correlated with $B R C A$ abnormality are not specific for germline $B R C A 1$ mutation and may not be informative about the presence of BRCA2 mutations. Also, we included 58 study cases that were known to lack germline BRCA 1 and 2 mutations, but the somatic mutation and BRCA1 methylation status was unknown. We had expected several of these cases to show BRCA-like metastatic patterns as it is estimated that BRCA1 and 2 somatic mutations and 
BRCA1 promoter methylation should be detectable in $\sim 15 \%$ of unselected ovarian and fallopian tube high-grade serous carcinomas. This relatively small group lacking germline BRCA mutations may therefore not be representative of otherwise unselected high-grade serous carcinomas. Although we discuss the relative advantages and disadvantages of three morphologically based methods for BRCA risk determination, we hesitate to formally compare the sensitivity and specificity of the current system with the others because, unlike the studies detailing primary site morphology and BRCA1 immunohistochemistry, the current study did not contain a validation cohort. Nevertheless, the features described here appear specific for the detection of high-grade serous carcinomas with homologous recombination deficiency, which can be therapeutically targeted with poly adenosine diphosphateribose polymerase inhibitors.

In summary, we demonstrate that metastatic BRCA-high-grade serous carcinomas exhibit characteristic morphological features that appear more sensitive and specific for BRCA abnormality than two other morphologically based prediction systems and are theoretically easier to apply in practice. These findings should be validated prospectively in an independent cohort.

\section{Disclosure/conflict of interest}

The authors declare no conflict of interest.

\section{References}

1 Tisserand P, Fouquet C, Barrois M, et al. Lack of HIN-1 methylation defines specific breast tumor subtypes including medullary carcinoma of the breast and BRCA1-linked tumors. Cancer Biol Ther 2003;2:559-563.

2 Armes JE, Egan AJM, Southey MC, et al. The histologic phenotypes of breast carcinoma occurring before age 40 years in women with and without BRCA1 or BRCA2 germline mutations: a population-based study. Cancer 1998;83:2335-2345.

3 Lakhani SR, Gusterson BA, Jacquemier J, et al. The pathology of familial breast cancer: histological features of cancers in families not attributable to mutations in BRCA1 or BRCA2. Clin Cancer Res 2000;6:782-789.

4 Wong EM, Southey MC, Fox SB, et al. Constitutional methylation of the BRCA1 promoter is specifically associated with BRCA1 mutation-associated pathology in early-onset breast cancer. Cancer Prev Res 2011; 4:23-33.

5 Soslow RA, Han G, Park KJ, et al. Morphologic patterns associated with BRCA1 and BRCA2 genotype in ovarian carcinoma. Mod Pathol 2012;25:625-636.
6 Fujiwara M, McGuire VA, Felberg A, et al. Prediction of BRCA1 germline mutation status in women with ovarian cancer using morphology-based criteria: identification of a BRCA1 ovarian cancer phenotype. Am J Surg Pathol 2012;36:1170-1177.

7 Kommoss F, Kommoss S, Schmidt D, et al. Survival benefit for patients with advanced-stage transitional cell carcinomas vs. other subtypes of ovarian carcinoma after chemotherapy with platinum and paclitaxel. Gynecol Oncol 2005;97:195-199.

8 Verhaak RG, Tamayo P, Yang JY, et al. Prognostically relevant gene signatures of high-grade serous ovarian carcinoma. J Clin Invest 2013;123:517-525.

9 Karamurzin Y, Leitao MM Jr., Soslow RA. Clinicopathologic analysis of low-stage sporadic ovarian carcinomas: a reappraisal. Am J Surg Pathol 2013;37: 356-367.

10 Gilks CB, Ionescu DN, Kalloger SE, et al. Tumor cell type can be reproducibly diagnosed and is of independent prognostic significance in patients with maximally debulked ovarian carcinoma. Hum Pathol 1 2008;39:1239-1251.

11 Soslow RA. Histologic subtypes of ovarian carcinoma: an overview. Int J Gynecol Pathol. I 2008;27: 161-174.

12 Ali RH, Seidman JD, Luk M, et al. Transitional cell carcinoma of the ovary is related to high-grade serous carcinoma and is distinct from malignant brenner tumor. Int J Gynecol Pathol 2012;31:499-506.

13 Takeuchi $\mathrm{T}$, Ohishi $\mathrm{Y}$, Imamura $\mathrm{H}$, et al. Ovarian transitional cell carcinoma represents a poorly differentiated form of high-grade serous or endometrioid adenocarcinoma. Am J Surg Pathol 2013;37: 1091-1099.

14 Garg K, Levine DA, Olvera N, et al. BRCA1 immunohistochemistry in a molecularly characterized cohort of ovarian high-grade serous carcinomas. Am J Surg Pathol 2013;37:138-146.

15 Hyman DM, Long KC, Tanner EJ, et al. Outcomes of primary surgical cytoreduction in patients with BRCAassociated high-grade serous ovarian carcinoma. Gynecol Oncol 2012;126:224-228.

16 du Bois A, Reuss A, Pujade-Lauraine E, et al. Role of surgical outcome as prognostic factor in advanced epithelial ovarian cancer: a combined exploratory analysis of 3 prospectively randomized phase 3 multicenter trials: by the Arbeitsgemeinschaft Gynaekologische Onkologie Studiengruppe Ovarialkarzinom (AGO-OVAR) and the Groupe d'Investigateurs Nationaux Pour les Etudes des Cancers de l'Ovaire (GINECO). Cancer 2009;115:1234-1244.

17 Winter WE 3rd, Maxwell GL, Tian C, et al. Tumor residual after surgical cytoreduction in prediction of clinical outcome in stage IV epithelial ovarian cancer: a Gynecologic Oncology Group Study. J Clin Oncol 2008;26:83-89.

18 Bristow RE, Tomacruz RS, Armstrong DK, et al. Survival effect of maximal cytoreductive surgery for advanced ovarian carcinoma during the platinum era: a meta-analysis. J Clin Oncol 2002;20:1248-1259. 